\title{
Brillouin Scattering Spectrum Analysis Based on Auto-Regressive Spectral Estimation
}

\author{
Mengyun HUANG, Wei LI, Zhangyun LIU, Linghao CHENG*, and Bai-Ou GUAN \\ Guangdong Provincial Key Laboratory of Optical Fiber Sensing and Communications, Institute of Photonics \\ Technology, Jinan University, Guangzhou, 510632, China \\ *Corresponding author: Linghao CHENGＥ-mail: chenglh@ieee.org
}

\begin{abstract}
Auto-regressive (AR) spectral estimation technology is proposed to analyze the Brillouin scattering spectrum in Brillouin optical time-domain refelectometry. It shows that AR based method can reliably estimate the Brillouin frequency shift with an accuracy much better than fast Fourier transform (FFT) based methods provided the data length is not too short. It enables about 3 times improvement over FFT at a moderate spatial resolution.
\end{abstract}

Keywords: Brillouin optical time-domain reflectometry (BOTDR); auto-regressive (AR) model; spectral estimation; distributed fiber-optic sensing

Citation: Mengyun HUANG, Wei LI, Zhangyun LIU, Linghao CHENG, and Bai-Ou GUAN, "Brillouin Scattering Spectrum Analysis Based on Auto-Regressive Spectral Estimation," Photonic Sensors, 2018, 8(2): 114-118.

\section{Introduction}

Brillouin scattering in optical fibers is sensitive to temperature and strain. Therefore, Brillouin scattering based distributed fiber-optic sensing system is very promising for wide range applications [1]. Brillouin optical time-domain reflectometry (BOTDR) and Brillouin optical time-domain analyzer (BOTDA) are two widely employed schemes. Normally, BOTDR is of interest in practical implementation due to its single-end launch and receiving configuration, making it more robust in events of fiber fracture.

To recover temperature and strain information from Brillouin scattering signal in BOTDR, one has to obtain the distribution of Brillouin frequency shift along the fiber, which can be done by spectral analysis of Brillouin scattering signal to find the frequency of the peak of the Brillouin gain spectrum. In many schemes, a frequency scanning configuration is employed to find this Brillouin frequency shift by measuring the signal strength at various frequency points one by one. Obviously, such schemes take very long time to finish one measurement because tens of frequency points have to be measured [2]. To shorten the measurement time, broadband schemes can be used by receiving all spectra of the Brillouin scattering signal followed by spectral analysis through algorithms. Fast Fourier transform (FFT) is a popular algorithm in spectral analysis of Brillouin gain spectrum (BGS). The FFT technique utilizes a wide-bandwidth photodetector and a high speed analog-to-digital converter to acquire the Brillouin signal in a single measurement. Then a section of successive signal is extracted out by a window with time length $T$ and is transformed

Received: 23 December 2017 / Revised: 1 March 2018

(C) The Author(s) 2018. This article is published with open access at Springerlink.com

DOI: 10.1007/s13320-018-0488-7

Article type: Regular 
into the frequency domain with the FFT arithmetic. The BGS for the corresponding fiber section is therefore obtained. Afterwards, the window slides with a step of $\Delta T$, and the FFT is performed continuously for the extracted signals at different time, which results in the evolution of BGS along with time. Because BGS at different positions show up at different time points in the received signal, the evolution of BGS along with time gives the BGS at different positions. However, to enhance the spatial resolution of BOTDR, one has to use data as few as possible, which results in great degradation of spectral resolution and hence temperature and strain resolution through FFT. Of course one can pad zero to data of short length to increase the spectral resolution through FFT, which is a widely adopted method in FFT-based schemes. The cost of this method is highly computational burden and huge throughput requirement between computational unit and data storage unit. The overall measurement speed is therefore seriously degraded. Currently, FFT-based schemes can achieve high resolution in spatial and in accuracy. However, the measurement speed remains a problem [3-7].

In this paper, we propose an auto-regressive (AR) model based algorithm to estimate Brillouin gain spectrum. In particular, Burg algorithm is used. It shows that the proposed AR-based Brillouin spectral estimation is reliable in estimating the Brillouin frequency shift distribution at a resolution better than FFT based methods, especially when the data length is relatively short to achieve a high spatial resolution at a fairly lower computational burden [8].

\section{Theory}

Physically, Brillouin scattering is initiated by Langevin noise source that describes the thermal excitation of acoustic waves [1]. Langevin noise is essentially a white noise. However, due to the geometry and the material characteristics of optical fibers, the response of optical fibers to acoustic waves is highly frequency dependent which shapes the spectrum of Langevin noise and manifests as Brillouin gain spectrum. The above process can be modeled as

$$
\phi(\omega)=\left|\frac{B(\omega)}{A(\omega)}\right|^{2} \sigma^{2}
$$

where $\sigma^{2}$ is a positive scalar standing for Langevin noise source, and $|B(\omega) / A(\omega)|^{2}$ represents the Brillouin gain spectrum. Equation (1) shows that Brillouin scattering can be viewed as a rational filter with a transfer function $B(\omega) / A(\omega)$ to filtering white noise of power $\sigma^{2}$ to produce Brillouin scattering signal [2]. This filtering process as shown by (1) can be written in the time domain as

$$
y(t)=\frac{B(z)}{A(z)} e(t)
$$

where $y(t)$ is the filter output, i.e. Brillouin scattering signal, and $e(t)$ is white noise with variance of $\sigma^{2} . z^{-1}$ is the unit delay operator. Therefore, the problem of Brillouin spectral estimation can be reduced to a problem of signal modeling by finding $A(z)$ and $B(z)$. For a rational filter, $A(z)$ and $B(z)$ can be written as

$$
\begin{aligned}
& A(z)=1+a_{1} z^{-1}+\ldots+a_{n} z^{-n} \\
& B(z)=1+b_{1} z^{-1}+\ldots+b_{m} z^{-m}
\end{aligned}
$$

where $a_{n}$ and $b_{m}$ are the $n$-th and $m$-th constant coefficients of $A(z)$ and $B(z)$, respectively. A signal $y(t)$ described by (2) is an auto-regressive moving average (ARMA) signal. It has been shown that the covariance of an ARMA signal satisfies the following expression [2]:

$$
r(k)+\sum_{i=1}^{n} a_{i} r(k-i)=0
$$

for $k>m$ where $r(k)$ is the $k$-th order autocorrelation coefficient of $y(t)$. With an assumption of $B(z)=1$ and hence $m=0$, the signal is assumed to be an auto-regressive (AR) signal, and (2) can be rewritten as follows:

$$
A(z) y(t)=e(t) .
$$

The covariance of an AR signal then satisfies (4) 
for all positive $k$ and for $k=0$, it can be written as

$$
r(0)+\sum_{i=1}^{n} a_{i} r(-i)=\sigma^{2} .
$$

Equations (4) and (6) give the following system of linear equations

$$
\left[\begin{array}{cccc}
r(0) & r(-1) & \cdots & r(-n) \\
r(1) & r(0) & & \vdots \\
\vdots & & \ddots & \\
r(n) & \cdots & & r(0)
\end{array}\right]\left[\begin{array}{c}
1 \\
a_{1} \\
\vdots \\
a_{n}
\end{array}\right]=\left[\begin{array}{c}
\sigma^{2} \\
0 \\
\vdots \\
0
\end{array}\right] .
$$

$\left[\begin{array}{lll}a_{1} & \cdots & a_{n}\end{array}\right]$ can then be solved from (7) provided autocorrelation coefficients of $y(t)$ are obtained which can be deduced from measured data. With $\left[\begin{array}{lll}a_{1} & \cdots & a_{n}\end{array}\right]$ obtained, $A(z)$ is also known, and hence the Brillouin spectrum can be estimated from (1).

For AR-model based spectral estimation, the resulted filter is an all-pole filter, making the model good at estimating spectrum with peaks. For broadband receiving schemes, the spectrum of Brillouin scattering signal is composed of narrow peaks in a broadband spectrum. Therefore, AR-model can well estimate Brillouin gain spectrum. There are several algorithms to solve (7) such as Yule-Walker method and least square method. In this paper, the maxium entropy method, also known as Burg algorithm, is employed because it results in more reliable estimation of autocorrelation coefficients, especially when data length is short [8].

\section{Experiments}

Figure 1 illustrates the BOTDR experimental setup. A coherent detection scheme is adopted. The laser is operating at $1550.12 \mathrm{~nm}$ with a linewidth less than $100 \mathrm{kHz}$. Its output is split into two branches with one for pulse generation through an optical switch based on a semiconductor optical amplifier (SOA) and the other as local light for coherent detection at the balanced detector. The pulse generated by the SOA switch is amplified by an erbium-doped fiber amplifier (EDFA) before injecting into the test fiber. The Brillouin scattering signal from the test fiber is directed to the balanced photodetector for coherent detection after amplified by another EDFA. The resulted signal after the balanced photodetector is centered at around $10.8 \mathrm{GHz}$ which is downcoverted to less than $500 \mathrm{MHz}$ by a $10.5 \mathrm{GHz}$ local oscillator. The downconverted Brillouin scattering signal is then acquired at $1 \mathrm{GSps}$ by data acquisition for digital processing. The test fiber is $2-\mathrm{km}$ long with one section of about $400 \mathrm{~m}$ placed in hot water to tune its temperature. The width of the optical pulse injected into the test fiber is $250 \mathrm{~ns}$. The acquired data in time domain corresponding to the heated section are extracted for analysis. Burg algorithm derived from AR-model is employed in comparison to the results by FFT. Brillouin frequency shift is obtained by finding the frequency of the spectrum peak [1].

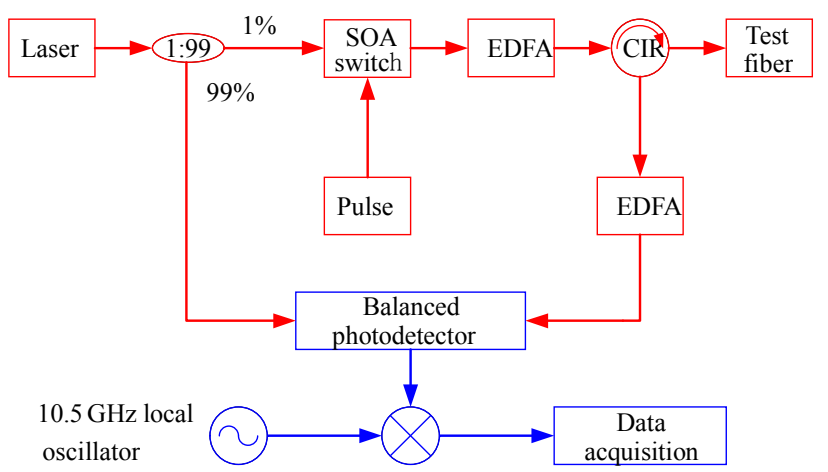

Fig. 1 BOTDR experimental setup. SOA: semiconductor optical amplifier; EDFA: erbium-doped fiber amplifier; CIR: circulator.

Figure 2 shows the calculated Brillouin frequency shift by Burg and FFT algorithms for several temperatures to compare the accuracies of the two methods. 1024 consecutive samples are used by algorithms for analysis. For each temperature, the measured data is averaged over 2500 pulses. Both FFT and Burg algorithms exhibit excellent linearity along with temperature. The slopes obtained by the two methods are very similar, $1.03167 \mathrm{MHz} /{ }^{\circ} \mathrm{C}$ for FFT and $1.032 \mathrm{MHz} /{ }^{\circ} \mathrm{C}$ for Burg. However, Burg shows better accuracy than FFT. The residual sum of squares is 0.753 for Burg while it is 2.476 for FFT. 


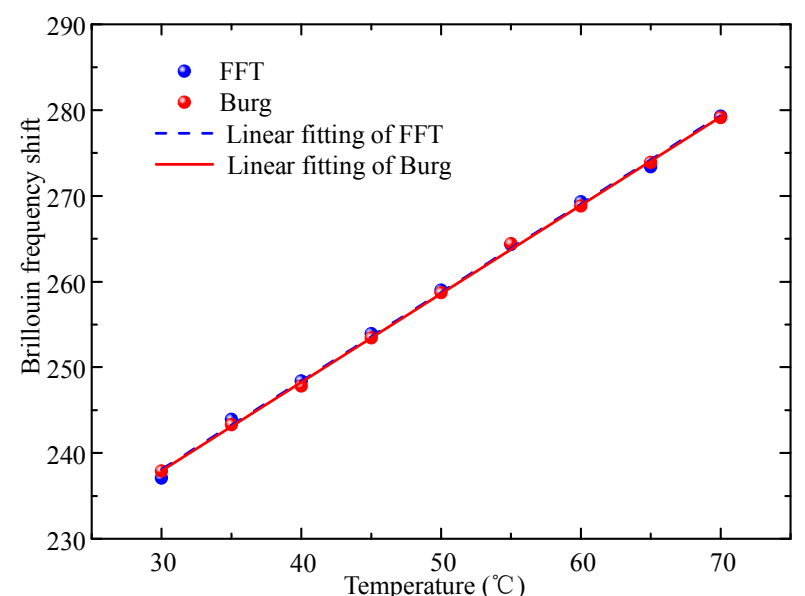

Fig. 2 Calculated Brillouin frequency shift by Burg and FFT algorithms for several temperatures.

Standard deviation is an important indicator of resolution of a measurement. Figure 3 shows the standard deviation resulted by FFT and Burg algorithms based on calculations on 1024 consecutive samples. To calculate standard deviations, 2500 pulses are grouped into 10 groups with 250 pulses in each group. Each group results in one measurement of Brillouin frequency shift and standard deviations are calculated based on these 10 measurements. Figure 3 shows that Burg results in much better standard deviation than that of FFT. Standard deviation is highly related to the spectral resolution. For FFT of 1024 point at $1 \mathrm{GSps}$, the frequency resolution is about $1 \mathrm{MHz}$. Therefore, the standard deviation of FFT is about $2 \mathrm{MHz}$. For Burg algorithm, standard deviation is dependent on the accuracy of the estimation of autocorrelation coefficients. With 1024 samples, this estimation is quite good and hence leads to a better standard deviation. However, when data length becomes short, the accuracy of the estimation of autocorrelation coefficients degrades, and worse standard deviation will be expected. Figure 4 shows this point for calculations on 32 consecutive samples. The standard deviation of Burg algorithm is worse than that of FFT. Note that because only 32 samples are used, they are padded by zeros to 1024 samples in FFT to achieve a spectral resolution of $1 \mathrm{MHz}$. The standard deviation of FFT is still around $2 \mathrm{MHz}$ while it is nearly doubled for Burg algorithm. Figure
5 illustrates this tendency more clearly by calculating the standard deviation of both algorithms for various lengths of consecutive samples used for calculations. For FFT, the data length is always padded to 1024 points. Therefore, the spectral resolution of FFT is always $1 \mathrm{MHz}$, and the standard deviation is basically flat at about $2 \mathrm{MHz}$ over all of these data lengths. For Burg algorithm, the standard deviation is better than that of FFT when data length is longer than 128. Shorter data length results in more unreliable autocorrelation coefficient estimation and hence leads to worse standard deviation. Therefore, Burg algorithm derived from AR-model can improve measurement accuracy over FFT-based algorithms provided the data length is not too short.

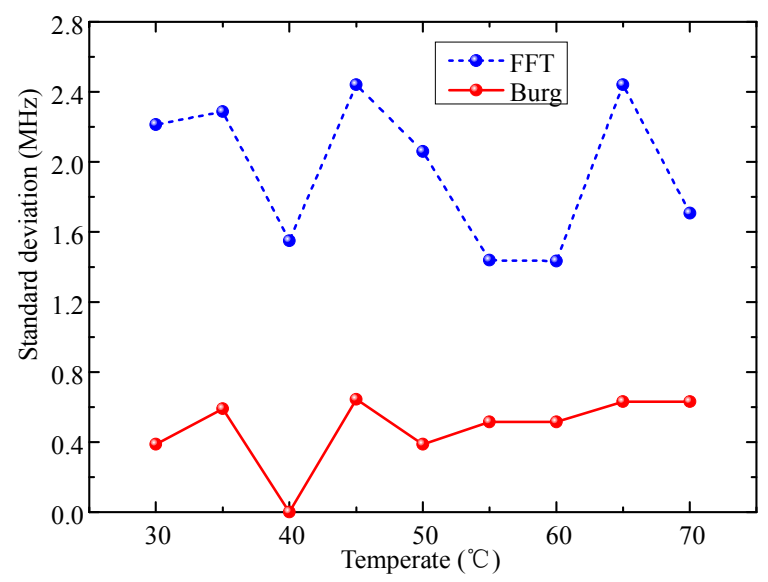

Fig. 3 Standard deviation resulted by FFT and Burg algorithms based on calculations on 1024 consecutive samples.

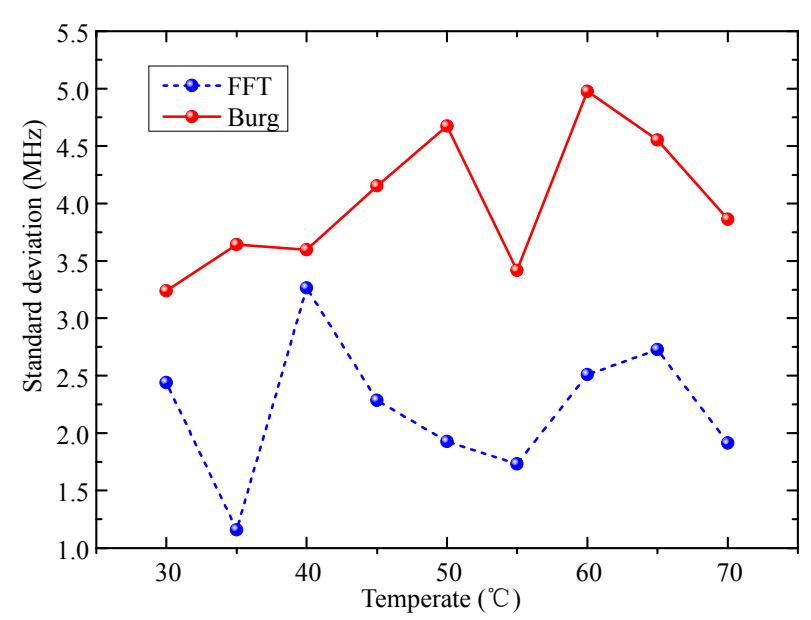

Fig. 4 Standard deviation resulted by FFT and Burg algorithms based on calculations on 32 consecutive samples. 


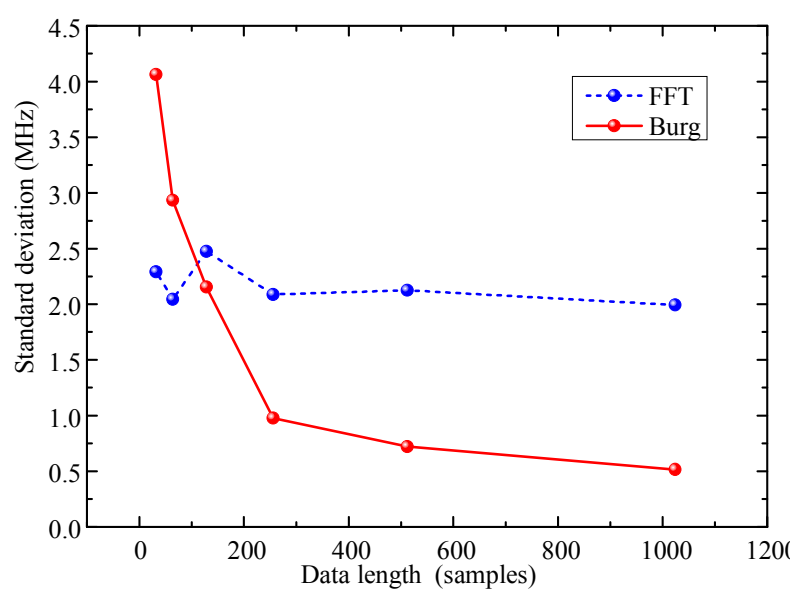

Fig. 5 Standard deviation resulted by FFT and Burg algorithms based on calculations at various lengths of consecutive samples.

\section{Conclusions}

Burg algorithm derived from AR-model is employed to analyze Brillouin scattering spectrum in the BOTDR system. Compared with FFT-based algorithms, Burg algorithm can improve measurement accuracy by lowering measurement standard deviation provided the data length is not too short. When the data length is longer than 128 samples, Burg algorithm outperforms FFT in our experiments. It then shows that Burg algorithm enables high accuracy measurement at a moderate spatial resolution.

\section{Acknowledgment}

This work was supported by the National Natural Science Foundation of China (Grant Nos. 11474133 and 61235005) and Science and Technology Program of Guangzhou (No. 201707010338).

Open Access This article is distributed under the terms of the Creative Commons Attribution 4.0 International License (http://creativecommons.org/ licenses/by/4.0/), which permits unrestricted use, distribution, and reproduction in any medium, provided you give appropriate credit to the original author(s) and the source, provide a link to the Creative Commons license, and indicate if changes were made.

\section{References}

[1] A. Yeniay, J. M. Delavaux, and J. Toulouse, "Spontaneous and stimulated Brillouin scattering gain spectra in optical fibers," Journal of Lightwave Technology, 2002, 20(8): 1425-1432.

[2] P. Stoica and R. Moses, Spectral analysis of signals. New Jersey, USA: Prentice Hall, 2005: 1-452.

[3] J. H. Geng, S. Staines, M. Blake, and S. B. Jiang, "Distributed fiber temperature and strain sensor using coherent radio-frequency detection of spontaneous Brillouin scattering," Applied Optics, 2007, 46(23): 5928-5932.

[4] F. Wang, X. P. Zhang, Y. G. Lu, R. R. Dou, and X. Y. Bao, "Spatial resolution analysis for discrete Fourier transform-based Brillouin optical time domain reflectometry," Measurement Science \& Technology, 2009, 20(2): 025202-1-025202-6.

[5] G. J. Tu, X. P. Zhang, Y. X. Zhang, Z. F. Ying, and L. D. Lu, "Strain variation measurement with shorttime Fourier transform-based Brillouin optical time-domain reflectometry sensing system," Electronics Letters, 2014, 50(22): 1624-1626.

[6] Y. F. Yu, L. Q. Luo, B. Li, L. F. Guo, J. Z. Yan, and K. Soga, "Double peak-induced distance error in short-time-Fourier-transform-Brillouin optical time domain reflectometers event detection and the recovery method," Applied Optics, 2015, 54(28): E196-E202.

[7] F. Wang, C. H. Zhu, C. Q. Cao, and X. P. Zhang, "Enhancing the performance of BOTDR based on the combination of FFT technique and complementary coding," Optics Express, 2017, 25(4): 3504-3513.

[8] F. Laeri, "Some comments on maximum entropy spectral analysis of time series," Computers in Physics, 1990, 4(6): 627-636. 